\title{
Design and Development of an in Vitro Assay for Evaluation of Solid Vaginal Dosage Forms
}

\author{
Jyoti Gupta ${ }^{1}$, Jason Qihai Tao ${ }^{2}$, Sanjay Garg ${ }^{1}$, Raida Al-Kassas ${ }^{{ }^{*}}$ \\ ${ }^{1}$ School of Pharmacy, Faculty of Medical and Health Sciences, The University of Auckland, Auckland, New Zealand; ${ }^{2}$ International \\ Partnership for Microbicides, Silver Springs, USA. \\ Email: *r.al-kassas@auckland.ac.nz
}

Received February $27^{\text {th }}, 2011$; revised April $11^{\text {th }}, 2011$; accepted July $20^{\text {th }}, 2011$.

\begin{abstract}
Vaginal dosage forms are seen as a viable option for empowering women to protect themselves from the risk of HIV transmission. Because of limited research in the field, there is a lack of suitable dissolution methods established for determination of drug release from vaginal formulations inside the vaginal tract. The main aim of this study was to develop a simple, reliable and reproducible in vitro release method for evaluation of solid vaginal dosage forms (VDFs) which was hoped to exhibit a close in vitro-in vivo correlation. Dapivirine, a drug being developed as a microbicide and a well established marketed anti fungal drug, Clotrimazole were used as model drugs. Two doses (0.5 mg and 1.25 $\mathrm{mg}$ ) of Dapivirine were prepared as novel rapidly disintegrating, bioadhesive tablets. Clotrimazole $100 \mathrm{mg}$, prepared in house as conventional release tablets and commercially available Canesten (Clotrimazole tablet $100 \mathrm{mg}$ ) were used. The in vitro drug release testing of these tablets was carried out using a designed system which consisted of modified USP dissolution Apparatus II in conjunction with Enhancer cell (as sample holder) in $150 \mathrm{ml}$ capacity flasks instead of the standard $900 \mathrm{ml}$ flasks. The suitability of the system was investigated for variable parameters such as formulation types, drug concentration, stirring speeds, media volume and comparison of in house product with marketed product. The method was successfully optimized at a volume of $100 \mathrm{ml}$ and a low speed of $25 \mathrm{rpm}$ at pH 4 and was found sensitive enough to distinguish between formulations and evaluate products of different strengths. A linear drug release profile $\left(R^{2}=0.99\right)$ was obtained in case of Dapivirine, indicating that drug release is controlled by diffusion. The developed dissolution system has a potential to exhibit a good in vitro-in vivo correlation in addition to carrying out routine dissolution tests for solid VDFs.
\end{abstract}

Keywords: Vaginal Solid Dosage Forms, Enhancer Cell, Dapivirine, Clotrimazole

\section{Introduction}

Administration of drugs in the vagina is believed to be as old as pharmacotherapy, first written documents dating from 19th century BC [1]. Vaginal drug delivery presents several advantages including, ease of administration, possibility of self-administration, hepatic first pass-effect bypass, low systemic drug exposure and increased permeability for some drugs when compared to the oral or other routes [1]. Vaginal dosage forms available around the world include creams, gels, tablets, capsules, pessaries, foams, ointments, films, tampons, rings and douches. While majority of vaginal drugs so far have been in the form of gels, there is a growing interest in alternative dosage forms such as tablets, rings, and films. Conventional vaginal dosage forms are associated with limitations of poor retention, leakage and messiness causing inconvenience to users, leading to poor subject/patient compliance and loss of therapeutic efficacy [2]. In recent years vaginal bioadhesive tablets have been developed as a new type of controlled release form for the treatment of both topical and systemic diseases. The greatest advantages of such bioadhesive tablets are the release of drug at a controlled rate and the possibility of maintaining them in the vagina for extended periods of time [3].

Dissolution testing is routinely used in Quality Control (QC) and Research and Development (R \& D). To date none of the official compendia [4-6] have included a standard method for evaluation of release pattern from vaginal preparations. Modified USP apparatus [7-10] and various other methods [11-13] have been employed by researchers to study the release of drugs from vaginal formulations. In these studies a standard dissolution apparatus with large volume of media has been employed 
which does not simulate the actual physiological conditions of the vagina, characterized by small volume of fluid. Volume plays a significant role in the rate of drug released and absorbed. In addition, other parameters such as speed of agitation and $\mathrm{pH}$ should be considered in order to assure close simulation of in vivo situation.

There are several systems used in the industry to study the release of drugs from various pharmaceutical dosage forms including vaginal formulations. These include USP apparatus 4 (Flow through cell), Franz cells and Enhancer cells $[14,15]$. Flow through cell could be used for solid and semi solid vaginal formulations; however the equipment is quite expensive and not available in many laboratories. On the other hand, Franz cell has been proposed to be one of the USP standard apparatus for topi$\mathrm{cal} /$ transdermal formulations. However, it is mostly suitable for semi-solid formulations and requires highly trained technician to operate it and a membrane to be a supportive physical barrier. The release of the drug from the formulation, in most instances, depends on the type of membrane to be used and its diffusion through it. Enhancer cell has also been used to evaluate release profiles of semi-solid formulations. The system consists of a paddle over enhancer cell with a small dissolution vessel. To date, most of the membranes used in this method were synthetic membranes or filters. This system utilizes the existing USP apparatus and is thus easy to set up, portable and reproducible.

In the present study, we have employed Enhancer cell system in conjunction with USP dissolution apparatus II and $150 \mathrm{ml}$ capacity flasks to replace the $900 \mathrm{ml}$ flasks used in the standard apparatus. The key difference of our design is "mesh membrane" which replaced the synthetic membranes most commonly used in past. The mesh membrane of adequate pore size holds the formulation in place and provide physical barrier which prevents the formulation from being agitated too much while allowing the drug to migrate or release freely from the formulation matrix to the media and allowing some contact/exchange of the media and the sample in the cavity. This system adopts physiological parameters of the vagina (volume of fluid, speed of agitation and $\mathrm{pH}$ ), in an attempt to obtain a standard quality control procedure for evaluation of vaginal tablets, to minimize variables in results and to achieve a close approximation to in vivo situation.

The aims of our design and the study were to develop a simple, reliable and reproducible in vitro release method for evaluation of solid vaginal dosage forms (VDFs) which mimicked in vivo vaginal application of a tablet dosage and was hoped to exhibit an adequate in vitro-in vivo correlation for future developments.

The drugs selected for the study were Dapivirine and Clotrimazole. Dapivirine, also known as TMC120, is a substituted diarylpyrimidine derivative and a non nucleoside reverse transcriptase inhibitor (NNRTI) being developed as a vaginal microbicide for prevention of HIV transmission [16]. Vaginal tablets of Dapivirine were prepared to disintegrate rapidly in the presence of small volume of fluid and form a bioadhesive gel from which the drug is slowly released over a period of time (there is no evidence yet for this bioadhesive tablet to be a controlled release formulation).

Clotrimazole is a chlorinated imidazole derivative widely prescribed for the treatment of vulvovaginal candidiasis VVC. Vaginal tablets of Clotrimazole were prepared in house as conventional release tablets.

\section{Materials and Methods}

\subsection{Materials}

Dapivirine was obtained as a gift from International Partnership for Microbicides (IPM). Clotrimazole was also received as a gift from Zhejiang Chemicals Import and Export Corporation, China. Canesten tablets (Clotrimazole tablets $100 \mathrm{mg}$ ) were procured from a local pharmacy in New Zealand. The directly compressible lactose was procured from Fonterra (New Zealand), microcrystalline cellulose (MCC) from Asahi Kasei Corp (Japan), magnesium stearate from Acros Organic (USA), sodium starch glycollate from Spectrum, USA. Ammonium acetate was purchased from BDH Laboratory (England), sodium phosphate dibasic from Riedel-de Haen (Germany), sodium phosphate monobasic, acetonitrile and methanol from Merck (Germany). Sodium lauryl sulphate was purchased from Serva Electrophoresis and tween 80 from Sigma Aldrich (Germany).

\subsection{Tablet Preparation}

Dapivirine was prepared in two different doses $(0.05 \%$ and $0.125 \%$ ) as novel rapidly disintegrating, long acting bioadhesive tablets. Dapivirine being a low dose drug was mixed geometrically with polymer, diluents, superdisintegrant and acid buffering agents to ensure a homogeneous mixing of the drug with the excipients. The mixing was carried out in a pilot scale cube mixer attached to a multipurpose unit operator (Erweka, Germany). The detailed composition and manufacturing procedure for Dapivirine cannot be disclosed due to Intellectual Property reasons.

Clotrimazole $100 \mathrm{mg}$ conventional vaginal tablets were prepared as follows: Clotrimazole, passed through 60 mesh, was mixed with MCC (passed through 30 mesh) in the cube mixer for 30 minutes. Directly compressible grade lactose was passed through 30 mesh and added to the mix of Clotrimazole and MCC and mixed for one hour. Magnesium stearate and sodium starch glycollate 
after passing through 44 mesh were added to the above blend and mixed for additional 10 minutes.

The tablets of Dapivirine and Clotrimazole were prepared by direct compression technique and compressed using a single punch tablet press (Cadmach, India) with almond shaped $(23 \times 13 \mathrm{~mm})$ punch. The average weight of Dapivirine and Clotrimazole tablets were set as $1.0 \mathrm{gm}$. These tablets were characterized for various parameters including hardness, thickness, moisture content, disintegration time and drug content.

\subsection{Methods of Analysis}

The HPLC methods were developed for Dapivirine and Clotrimazole and validated for linearity, specificity, accuracy and precision according to ICH guidelines (ICH). Dapivirine was analysed using a Waters Symmetry Sheild $\mathrm{RP}_{18}$ column, $250 \mathrm{~mm} \times 4.6 \mathrm{~mm}$ (Waters, USA) and acetonitrile and $5 \mathrm{mM}$ Ammonium acetate buffer $(\mathrm{pH} 4.5)$ in the ratio of $80: 20 \mathrm{v} / \mathrm{v}$ as mobile phase with a detection wavelength of $286 \mathrm{~nm}$. Clotrimazole was analyzed using a Waters Nova Pack $\mathrm{C}_{18}$ column, $150 \times 3.9 \mathrm{~mm}$ (Waters, USA) and $5 \mathrm{mM}$ sodium dibasic phosphate buffer and methanol in a composition of $25: 75 \mathrm{v} / \mathrm{v}$ as mobile phase at a wavelength of $262 \mathrm{~nm}$.

\subsection{Solubility Studies}

Solubility studies of Dapivirine and Clotrimazole were carried out by shaking an excess amount of each drug with $3 \mathrm{ml}$ of the following dissolution medium simulated vaginal fluid, sodium phosphate buffer of $\mathrm{pH} 4$ and $1 \%$ Tween, sodium phosphate buffer $\mathrm{pH} 4$ and $0.5 \%$ sodium lauryl sulphate, sodium phosphate buffer $\mathrm{pH} 4$ and $1 \%$ sodium lauryl sulphate, sodium phosphate buffer $\mathrm{pH} 4$ and methanol (ratio 75:25), sodium phosphate buffer pH4 and methanol (ratio 60:40), sodium phosphate buffer $\mathrm{pH} 4$ and methanol (ratio 40:60). The samples were incubated in a $37^{\circ} \mathrm{C}$ water bath (JEIO TECH, Model: BS-06/11/21/31, Korea) at $80 \mathrm{rpm}$ for 24 hours. After 24 hours, the samples were centrifuged in Eppendorf centrifuge (Model $5810 \mathrm{R}$, Germany) at 10,000 rpm for 10 minutes. The supernatants were collected and filtered through $0.45 \mu \mathrm{m}$ filters and injected into HPLC for analysis.

\subsection{Dissolution Apparatus Design}

A USP 8-Flask/8-pindle SR8-Plus (Hanson Research, USA) dissolution tester was used. The USP dissolution Aperture was modified with a "small volume" adapter kit (p/n 72-800-721, Hanson Research), including $150 \mathrm{ml}$ flat-bottom flasks and mini paddles. The Enhancer Cell (p/n 12-4000, VanKel, USA) as shown in Figure 1, consisted of a cap, a washer-membrane, an O-ring, and an adjustable drug reservoir. Specified volume of the de- gassed in vitro release testing (IVRT) medium was placed into each of the six vessels and the medium was equilibrated to $37^{\circ} \mathrm{C} \pm 0.5^{\circ} \mathrm{C}$. Each tablet was placed in the Enhancer cell cavity. Residual volume of the cell cavity was filled with the medium $(\sim 2 \mathrm{ml})$. The cell cavity was immediately covered with nylon mesh, O-ring and secured with the cap. Each Enhancer cell was gently slid into each of the vessel and the apparatus was operated at the specified rotation speed. At each sampling point indicated in Table 1, a syringe was fitted to the stainless steel sampling probe to withdraw $2 \mathrm{ml}$ of solution from a zone midway between the surface of the medium and the top of the paddle, not less than $1 \mathrm{~cm}$ from the vessel wall. $2 \mathrm{ml}$ of the dissolution medium was replaced back to each vessel after every sampling point. The sample solution was filtered through a $13 \mathrm{~mm} \times 0.45 \mu \mathrm{m}$ nylon filter and collected into HPLC vial for analysis.

The method was optimized by studying different variables:

\subsubsection{Selection of Drugs}

The two drugs used in this study were Dapivirine and Clotrimazole. The selection of drugs was based on two parameters:

1) Developmental Stage of the drugs (Novel vs Established).

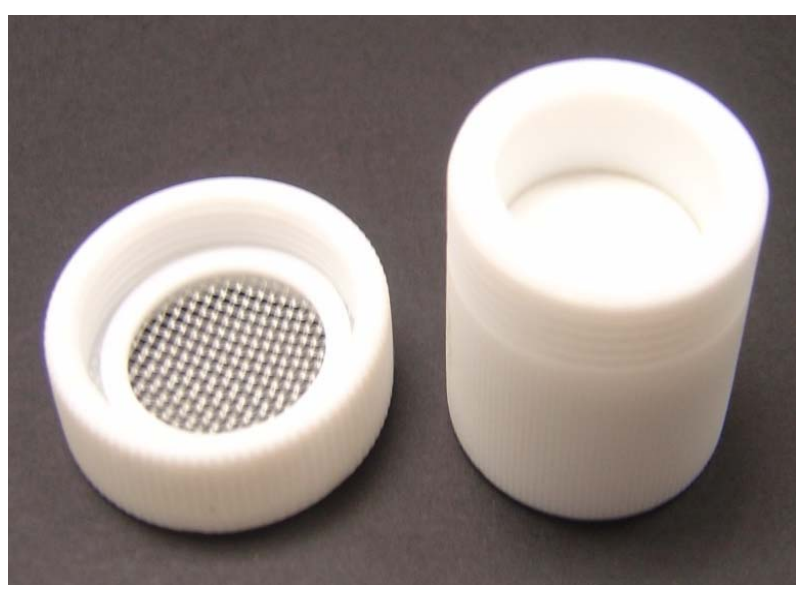

Figure 1. Enhancer cell.

Table 1. Dissolution conditions.

\begin{tabular}{ll}
\hline Media volume & $100 \mathrm{ml}, 150 \mathrm{ml}$ \\
Temperature & $37.0^{\circ} \mathrm{C} \pm 0.5^{\circ} \mathrm{C}$ \\
Paddle Height & $2.5 \mathrm{~cm}$ (Distance from paddle to membrane) \\
Paddle Rotation Speed & $100,75,50$ and $25 \mathrm{rpm}$ \\
Sampling volume & $2 \mathrm{ml}$ with replacement \\
Sampling Time Point & $0.25,0.5,1,2,3,4,6,8$ hours
\end{tabular}


Dapivirine is an investigational compound, being developed as a potential vaginal microbicide for the prevention of HIV transmission in women. Clotrimazole is a broad spectrum antimycotic agent widely used for vaginal candidiasis. It is a well established marketed product available in various dosage forms.

2) Formulation Design.

Dapivirine tablets have been prepared as novel rapidly disintegrating, bioadhesive tablets expected to be retained in the vaginal cavity for prolonged periods whereas, Clotrimazole tablets were prepared as conventional release tablets.

\subsubsection{In Vitro Release Testing Media}

Selection of the receptor medium was based on the solubility and stability of the drug in that medium. The receptor medium must provide adequate stability and sink conditions to ensure that the drug has sufficient solubility in the receptor medium such that it does not affect the release rate of the drug from the matrix. The vaginal $\mathrm{pH}$ of healthy women of reproductive age is acidic $(\mathrm{pH} 4-5)$ [17]. Hence sodium phosphate buffer $(\mathrm{pH} 4.0)$ was selected as the media. Dapivirine and Clotrimazole being poorly water soluble, solubility of these drugs were conducted in the selected media with different concentrations of surfactants and co solvents.

\subsubsection{Media Volume}

Two volumes were selected to study this parameter-100 $\mathrm{ml}$ and $150 \mathrm{ml}$. The drug selected for this study was Dapivirine $0.5 \mathrm{mg}$ tablets.

\subsubsection{Stirring Speed}

The tablets are not expected to undergo much agitation in the vagina due to weak contractions occurring in that area of the body. Hence, release studies of tablets were conducted at low speed $(25 \mathrm{rpm})$ and the results were compared with the release profiles obtained at higher speeds $(50,75,100)$ to determine if speed can have a significant effect on release rate of drugs. The drug selected for this study was Dapivirine $0.5 \mathrm{mg}$ tablets.

\subsubsection{Dose Variability}

The two doses of Dapivirine tablets $(0.5$ and $1.25 \mathrm{mg})$ were used to study the effect of concentration on the release properties of the drug. The tablets were prepared with the same manufacturing procedure, having similar formulation characteristics.

\subsubsection{Comparison of Prepared and Marketed Clotrimazole Tablets}

The drug release profile of Clotrimazole tablet $100 \mathrm{mg}$ prepared in house was compared with that of commercial Canesten (Clotrimazole tablet $100 \mathrm{mg}$ ) tablet, in order to establish the ability of the method to detect differences in the formulation characteristics.

\subsection{Kinetics of Drug Release}

To describe the kinetics of drug release from Dapivirine $(0.5 \mathrm{mg}$ and $1.25 \mathrm{mg})$ and Clotrimazole formulations, dissolution data obtained from drug release studies was fitted to various mathematical models such as zero-order, first order and Higuchi model. Korsmeyer-Peppas model was also applied to analyze the mechanism of drug release.

\section{Results and Discussion}

A well designed dissolution testing is important not only as a method for evaluation of a dosage form but also as a potential tool to predict the in vivo drug release behavior of the formulation. Currently, no official compendia specify a test to study the release pattern for vaginal formulations. The main aim of this study was to develop an in vitro dissolution procedure which is close to the physiological conditions of vagina thus to be able to give some predictive estimate of the in vivo drug release characteristics.

\subsection{Tablet Properties}

Tablets of Dapivirine and Clotrimazole were prepared successfully and complied with USP in respect to drug content $(98 \%-102 \%)$ and having the physical characteristics as described in Table 2. Dapivirine tablets, being fast dissolving, disintegrated within 60 seconds.

\subsection{Solubility Studies}

Selection of IVRT medium was based on physiological $\mathrm{pH}$ and solubility of the drug in that medium. In order to have good sink conditions, it is important to select a media in which the solubility of the drug is at least 3 to 10 times its saturation solubility. Solubility of Dapivirine and Clotrimazole was first determined in simulated vaginal fluid (SVF) prepared according to Owen and Katz [18]. However, low solubility results were observed due to hydrophobic nature of the drugs. Hence, the solubility of the drugs in phosphate buffer $(\mathrm{pH} 4)$ containing various surfactants and alcohol as co-solvents at different concentration was evaluated (Table 3).

\subsection{The Design}

Vaginal cavity usually contains very small volume of fluid [18]. The cavity of the enhance cell creates and mimics the situation when the formulation is placed inside the vaginal cavity as the tablet is allowed to disintegrate in small amount of fluid $(2 \mathrm{ml})$ inside the enhancer cell. Artificial membranes (synthetic and semi-synthetic) have been recommended for in vitro quality control testing 
Table 2. Physical characteristics of Dapivirine $0.5 \mathrm{mg}$ and $1.25 \mathrm{mg}$ and Clotrimazole $100 \mathrm{mg}$ tablets.

\begin{tabular}{lccc}
\hline \multicolumn{1}{c}{ Parameters } & Dapivirine $0.5 \mathrm{mg}$ & Dapivirine $1.25 \mathrm{mg}$ & Clotrimazole $100 \mathrm{mg}$ \\
\hline Average weight $(\mathrm{g})$ & $1.0175 \pm 0.003$ & $1.010 \pm 0.002$ & $1.009 \pm 0.12$ \\
Hardness (horizontal) (Kp) & $11.028 \pm 0.12$ & $11.25 \pm 0.42$ & $14.015 \pm 0.19$ \\
Hardness (vertical) (Kp) & $7.93 \pm 0.33$ & $8.07 \pm 0.50$ & $11.39 \pm 0.28$ \\
Thickness (mm) & $5.36 \pm 0.04$ & $5.39 \pm 0.01$ & $4.95 \pm 0.07$ \\
Friability (\% w/w) & 0.65 & 0.57 & 0.72 \\
Disintegration time (min) & $0.52 \pm 0.05$ & $0.58 \pm 0.03$ & $6.19 \pm 0.10$ \\
Moisture content (\% w/w) & $2.54 \pm 0.04$ & $2.51 \pm 0.02$ & $1.25 \pm 0.02$ \\
\hline
\end{tabular}

Table 3. Solubility data of Dapivirine and Clotrimazole in SVF and phosphate buffer containing surfactants and co-solvents.

\begin{tabular}{lcc}
\hline \multicolumn{1}{c}{ Media } & Concentration of Dapivirine $(\mu \mathrm{g} / \mathrm{ml})$ & Concentration of Clotrimazole $(\mu \mathrm{g} / \mathrm{ml})$ \\
\hline Simulated Vaginal Fluid & 0.53 & 21.30 \\
Sodium Phosphate Buffer $(\mathrm{pH} 4.0)+0.5 \%$ Tween 80 & 21.19 & 22.06 \\
Sodium Phosphate Buffer (pH 4.0)+ 1.0\% Tween 80 & 40.21 & 412.30 \\
Sodium Phosphate Buffer (pH 4.0) + 0.5\% SLS & 36.82 & 1338.60 \\
Sodium Phosphate Buffer (pH 4.0) + 1.0\% SLS & 123.51 & 16.82 \\
Sodium Phosphate Buffer (pH 4.0):Methanol (75:25) & 0.85 & 102.08 \\
Sodium Phosphate Buffer (pH 4.0):Methanol (60:40) & 7.18 & 2323.11 \\
Sodium Phosphate Buffer (pH 4.0):Methanol (40:60) & 112.70 & \\
\hline
\end{tabular}

due to the variability involved with biological membranes. The membranes should be permeable to the drug and its pore size and thickness should not affect the drug release. The membrane selected should provide an inert holding surface for the test formulation, and not act as a barrier as vaginal microbicidal formulations are not designed for systemic absorption but expected to act topically. The Nylon Mesh used during this entire experiment served only as a holder first for the placed tablet and then for the gel-matrix obtained after disintegration of the tablet in the sample compartment in contact with the receptor solution. The mesh size was small enough to hold disintegrated tablet inside the sample cavity but still permitted the receptor solution to easily circulate from the $150 \mathrm{ml}$ dissolution vessel into the sample cavity of the Enhancer cell, thus facilitating homogenous distribution of the drug released from the formulation.

\subsection{In Vitro Drug Release Studies}

\subsubsection{Selection of Media}

Dapivirine and Clotrimazole are poorly water soluble drugs. This necessitated the use of media with surfactant or organic solvent. Based on the solubility data of the drugs (Table 3), the two media selected to study the release profile were sodium phosphate buffer containing 1) $1 \%$ SLS and 2) $60 \%$ methanol. The release study was conducted at a paddle rotation speed of $100 \mathrm{rpm}$ and media volume was $100 \mathrm{ml}$. Figures 2 and $\mathbf{3}$ show comparative release profiles of Dapivirine and Clotrimazole, respectively in two media. Dapivirine release profile from surfactant medium showed a sharp release in 15 minutes, forming a plateau after 6 hours. However, profile in aqueous organic medium demonstrated a slow and linear rise in the drug release during the 8 hours study. This can be explained by higher solubility of Dapivirine in surfactant media $(123.5 \mu \mathrm{g} / \mathrm{ml})$ as compared to aqueous organic media $(112.7 \mu \mathrm{g} / \mathrm{ml})$. Due to high probability of air bubbles with the use of surfactant media, aqueous organic media which shows a profile closer to the expected in vivo drug release behavior was selected as the media of choice. Previous reports [19,20] on Dapivirine have used aqueous organic media for drug release studies. On the other hand, Clotrimazole release in aqueous organic media was higher as compared to that in surfactant media, which is attributed to the drug's higher solubility in aqueous organic media $(2323 \mu \mathrm{g} / \mathrm{ml})$. 


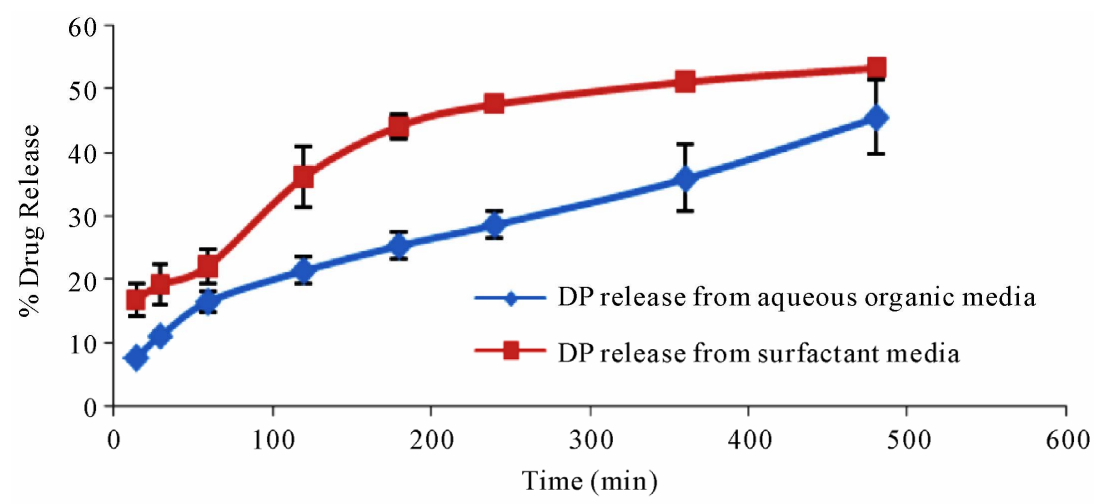

Figure 2. Dapivirine release profile in aqueous organic and surfactant media.

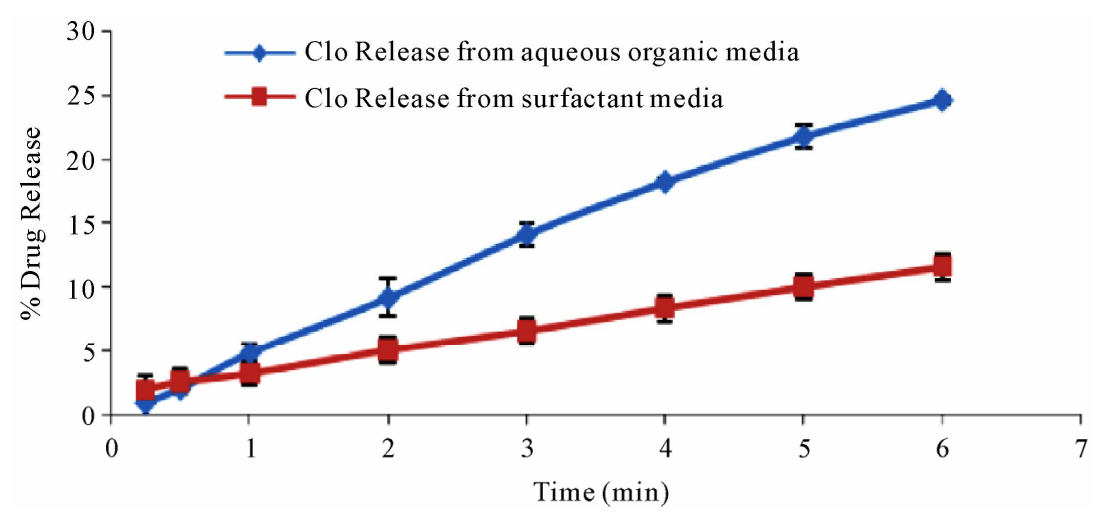

Figure 3. Clotrimazole release profile in aqueous organic and surfactant media.

\subsubsection{Media Volume}

Figure 4 shows the effect of the media volume on the release of Dapivirine tablets. The study was conducted using $100 \mathrm{ml}$ and $150 \mathrm{ml}$ of dissolution media. As can be seen from the Figure 4, the percentage drug release in the two volumes $(30.6 \%$ in $100 \mathrm{ml}$ and $27.4 \%$ in $150 \mathrm{ml}$ after 8 hours) was not markedly different from each other; $100 \mathrm{ml}$ was selected as the volume for further studies. Vaginal cavity usually contains very small volume of fluid [18]. The lowest volume in $150 \mathrm{ml}$ flask which could be selected was $100 \mathrm{ml}$. During conducting the dissolution studies, it is important that the sink condition is maintained. These parameters were considered in selection of the dissolution volume in our study. After 8 hours, Dapivirine release in $100 \mathrm{ml}$ of media was approximately $30 \%(1.6 \mu \mathrm{g} / \mathrm{ml})$, indicating that the sink conditions were maintained as the saturation solubility of Dapivirine in the receptor medium was 10 times higher than the maximum concentration detected during the 8 hours of drug release study. Thus with the sink conditions present, it is more likely that dissolution results will reflect the properties of the dosage form [6].

\subsubsection{Stirring Speed}

In order to simulate the physiological conditions of weak contractions in vagina, release rate of Dapivirine tablet was studied at the lowest allowable speed of rotation in a standard USP apparatus- $25 \mathrm{rpm}$. The percent drug release at 2 and 6 hours were compared for different speeds of rotation (Table 4). As can be seen from the table, maximum drug release was observed with $100 \mathrm{rpm}$ rotational speed. This can be attributed to the fact that an increase in agitation leads to a reduction in the thickness of the diffusion layer formed at the interface between the receptor and the membrane and facilitates better mixing [14]. The release at $100 \mathrm{rpm}$ was approximately 1.5 times higher than at $25 \mathrm{rpm}$. However, the tablets at $25 \mathrm{rpm}$ showed a comparable drug release rate to 50 or $75 \mathrm{rpm}$ and were closer to physiological conditions of weak contractions; therefore $25 \mathrm{rpm}$ was selected as the speed of choice.

\subsubsection{Comparison of Prepared and Marketed Clotrimazole Tablets}

This study was conducted to compare the release profiles of marketed Canesten tablets with the prepared Clotrimazole tablets. The drug release profiles in Figure 5 shows an initial higher release rate $(5.5 \%)$ of the drug from Canesten as compared to that from in house Clotrimazole tablets $(1.0 \%)$. This may be due to the variation 


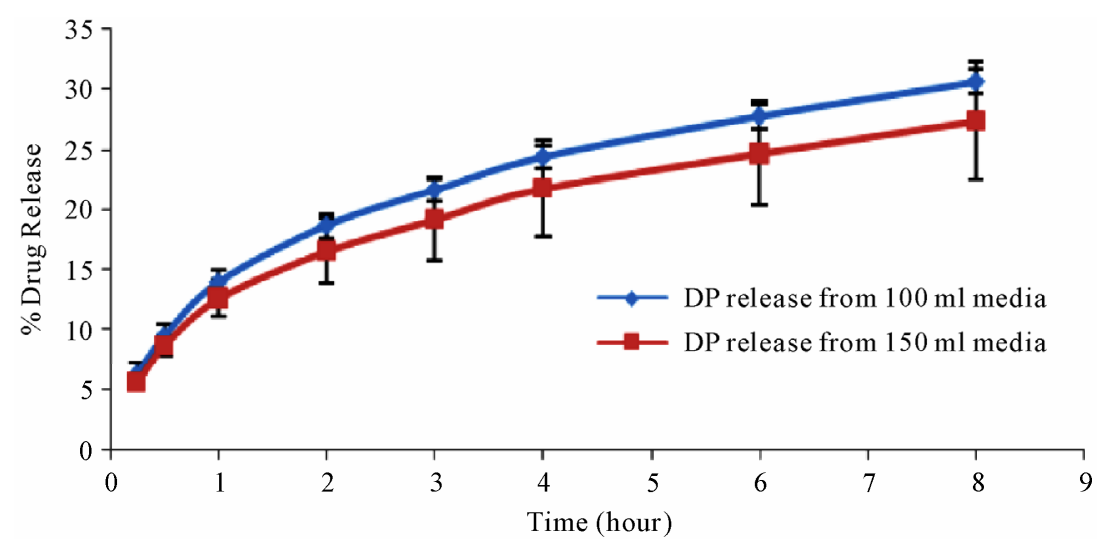

Figure 4. Comparison of Dapivirine release in different media volumes.

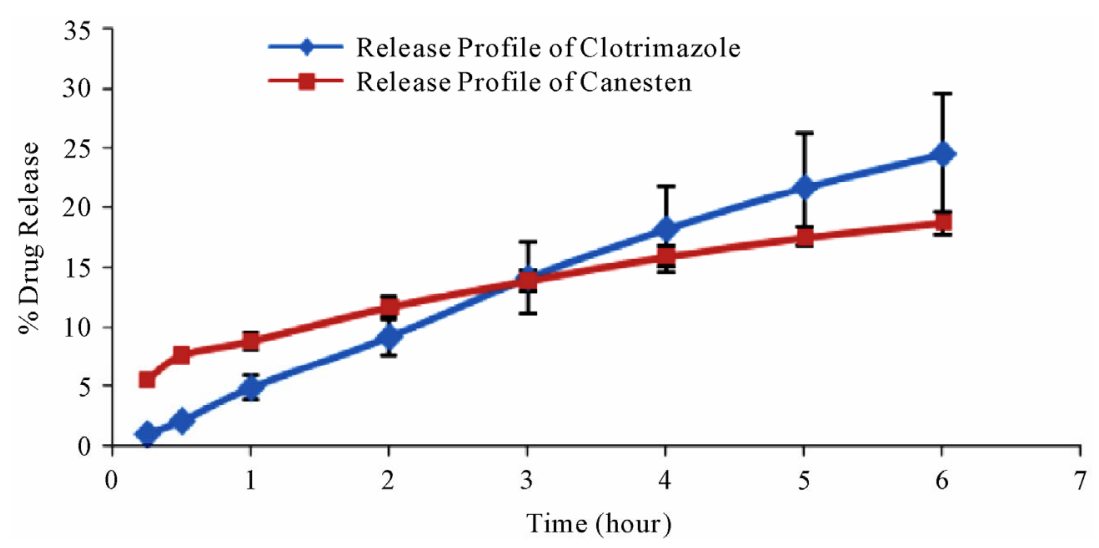

Figure 5. Release profile comparison of different formulations of Clotrimazole.

Table 4. Comparison of Dapivirine release after 2 and 6 hours at different speeds of rotation.

\begin{tabular}{ccc}
\hline $\begin{array}{c}\text { Paddle Speed } \\
(\mathrm{rpm})\end{array}$ & $\begin{array}{c}\text { Release in 2 hours } \\
(\%)\end{array}$ & $\begin{array}{c}\text { Release in 6 hours } \\
(\%)\end{array}$ \\
\hline 25 & 14.7 & 23.6 \\
50 & 20.2 & 29.9 \\
75 & 18.6 & 27.8 \\
100 & 21.6 & 36.0 \\
\hline
\end{tabular}

in the disintegration time of the two products. The disintegration time of the two formulations when measured using standard BP Disintegration apparatus, was found to be 6 - 7 minutes for prepared Clotrimazole tablets and 45 seconds for Canesten. However, after a period of time the release profiles of the marketed Canesten and the prepared clotrimazole tablets became comparable. Thus, it is evident that the method is sensitive enough to differentiate variability in the method of manufacturing.

\subsubsection{Dose Variability}

This parameter was based on release studies conducted on two different doses of Dapivirine $(0.05 \%$ and $0.125 \%)$, in order to assess the sensitivity of the developed dissolution method (Figure 6). The dissolution conditions were as reported in Table 1. From Figure 6, it can be seen that the shape of the two release profiles is similar showing a higher release from the higher dose, indicating the capability of the method to differentiate the variability in dose.

\subsection{Comparison of Release Profile from Enhancer Cell and Standard USP Dissolution Method}

The drug release study was conducted using the standard dissolution method described in official compendia [4-6] for solid oral dosage forms. The release profile from standard method was compared with that from Enhancer cell method. Dapivirine $0.5 \mathrm{mg}$ tablet was employed in this study and $500 \mathrm{ml}$ of $0.1 \mathrm{~N} \mathrm{HCL}$ was used as media. As can be seen from Figure 7, Enhancer cell method shows a slow release as only $20 \%$ of drug is released within 2 hours. On the other hand, the release rate of drug studied in standard dissolution apparatus is high as $80 \%$ of drug is released within 2 hours.

With the standard USP dissolution method, employing 


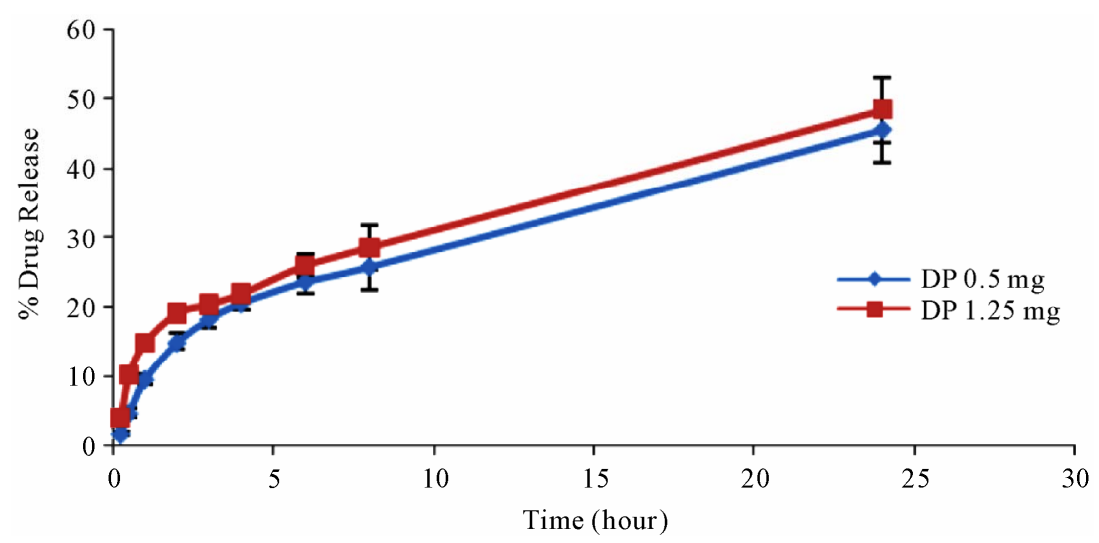

Figure 6. Comparison of Dapivirine release from two doses (0.5 $\mathrm{mg}$ and $1.25 \mathrm{mg})$.

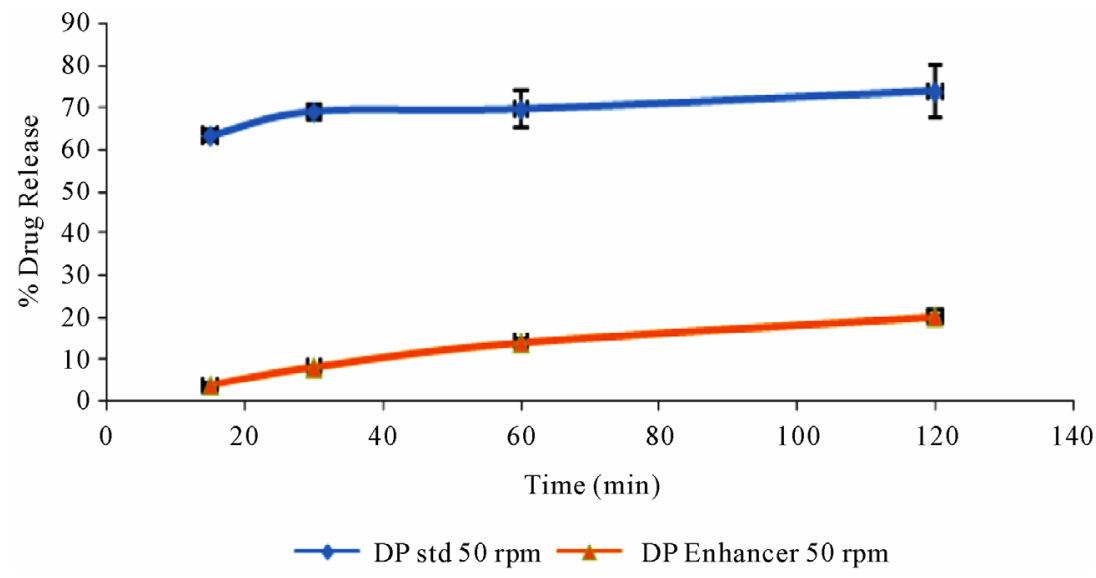

Figure 7. Comparative release profile of Dapivirine from standard and enhancer cell method at $50 \mathrm{rpm}$.

$500 \mathrm{ml}$ of media and $50 \mathrm{rpm}$ rotational speed, the rapidly disintegrating tablets of Dapivirine dispersed quickly in the large media volume giving a solubility profile of the drug rather than predicting its release characteristics. These tablets were designed to disperse quickly and form a gel in small amount of fluid in the vagina. Enhancer cell has the advantage of containing the tablet in a pocket wherein it is allowed to disperse in small volume $(2 \mathrm{ml})$ of media and form a bioadhesive gel from which the drug is released slowly. Comparative release studies of Dapivirine from Enhancer cell and standard method at 50 rpm clearly show that the shape of the two profiles is same. The only difference is the rate of release of the drug which corresponds to the difference in the volume.

\subsection{Kinetics and Mechanism of Drug Release}

The release data of Dapivirine $0.5 \mathrm{mg}$ and $1.25 \mathrm{mg}$ were fitted best to Higuchi's model $\left(\mathrm{R}^{2}=0.989\right.$ and 0.992 respectively) as indicated in Figures 8 and 9 . This suggests that the drug release is controlled by diffusion. When the release data was fitted to the model of Korsmeyer-Peppas (Table 5), the release exponent $n$ value was found to be close to 0.5 for both the doses thus indicating Fickian diffusion. The drug release data of Clotrimazole tablets was best fitted to zero-order model with an $\mathrm{R}^{2}$ value of 0.9957. This was confirmed when the data was applied to Korsmeyer Peppas model. The value of release exponent $n$ was found to be close to 1.0 (Table 5) indicating zeroorder release.

\section{Conclusions}

A dissolution system was designed and optimized for evaluation of vaginal tablets. The system enabled containment of the drug matrix in the cell to avoid complete distribution and dissolution of the drug in the medium. Drug release studies were successfully conducted on Dapivirine and Clotrimazole tablets. The suitability of the system was investigated for various parameters like different formulation design, dose variability, different stirring speeds, media volume and comparison of prepared and marketed product. The Enhancer cell method was found to be sensitive enough to distinguish between formulations and demonstrated the ability to detect products of different strengths. In conclusion, a simple, 


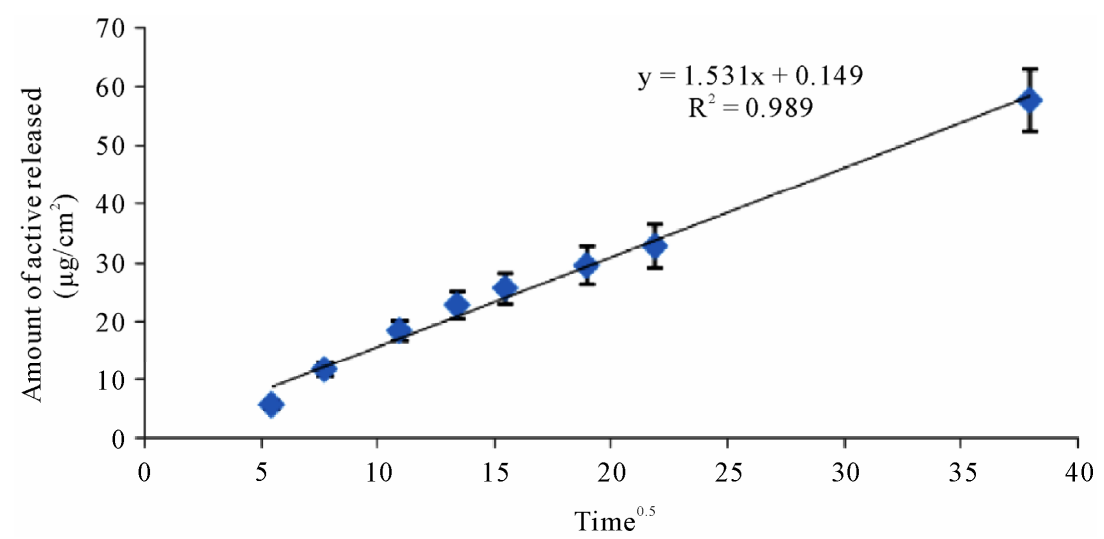

Figure 8. Dapivirine $0.5 \mathrm{mg}$ release per $\mathrm{cm}^{2}$ versus square root of time.

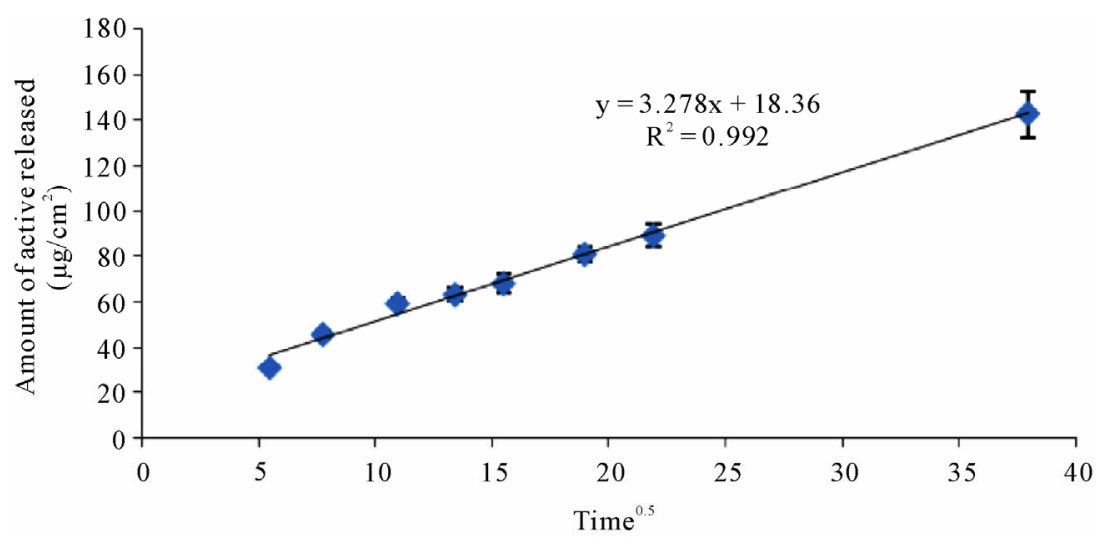

Figure 9. Dapivirine $1.25 \mathrm{mg}$ release per $\mathrm{cm}^{2}$ versus square root of time.

Table 5. Model fitting using Korsmeyer-Peppas equation.

\begin{tabular}{cccc}
\hline Product & $\mathrm{R}^{2}$ & $n$ & $k$ \\
\hline Dapivirine $0.5 \mathrm{mg}$ & 0.9272 & 0.6907 & 0.4090 \\
Dapivirine $1.25 \mathrm{mg}$ & 0.9161 & 0.4742 & 1.6420 \\
Clotrimazole $100 \mathrm{mg}$ & 0.9624 & 0.9122 & 0.0850 \\
\hline
\end{tabular}

reliable, and reproducible dissolution method has been developed for solid vaginal dosage forms. This method can be used to support formulation development for early stage drug product development and potentially for quality control and in vivo performance analysis of the final product.

\section{REFERENCES}

[1] J. Das Neves, "Vaginal Drug Delivery," SciTopics, 2008.

[2] J. Robinson and W. Bologna, "Vaginal and Reproductive System Treatments Using a Bioadhesive Polymer," Journal of Controlled Release, Vol. 28, No. 1-3, 1994, pp. 87-94. doi:10.1016/0168-3659(94)90156-2

[3] A. Gursoy and A. Bayhan, "Testing of Drug Release from Bioadhesive Vaginal Tablets," Drug Development and In- dustrial Pharmacy, Vol. 17, No. 18, 2007, pp. 2457-2475. doi:10.3109/03639049109048087

[4] European Pharmacopoeia 60: EDQM, 2007.

[5] British Pharmacopoeia, Stationary Office, London, 2009.

[6] United States Pharmacopoeia and National Formulary, 2009.

[7] A. El-Kamel, M. Sokar and V. Naggar, "Bioadhesive Controlled Release Metronidazole Vaginal Tablets," Acta Pharmaceutica, Vol. 52, 2002, pp. 171-179.

[8] S. Garg, L. Jambu and K. Vermani, "Development of Novel Sustained Release Bioadhesive Vaginal Tablets of Povidone Iodine," Drug Development and Industrial Pharmacy, Vol. 33, No. 12, 2007, pp. 1340-1349. doi: $10.1080 / 03639040701385782$

[9] F. Ahmad, M. Alam, Z. Khan, M. Khar and M. Ali, "Development and in Vitro Evaluation of an Acid Buffering Bioadhesive Vaginal Gel for Mixed Infections," Acta Pharmaceutica, Vol. 58, No. 4, 2008, pp. 407-19. doi:10.2478/v10007-008-0023-2

[10] L. Wang and X. Tang, "A Novel Ketoconazole Bioadhesive Tablet for Vaginal Delivery: Design, in Vitro and in Vivo Evaluation," International Journal of Pharmaceutics, Vol. 350, No. 1-2, 2008, pp. 181-187.

[11] C. Lee and Y. Chien, "Development and Evaluation of 
Mucoadhesive Drug Delivery System for Dualcontrolled Delivery of Nonoxynol-9," Journal of Controlled Release, Vol. 39, No. 1, 1996, pp. 93-103. doi:10.1016/0168-3659(95)00142-5

[12] C. Valenta, C. Kast and I. Harich, "Development and in Vitro Evaluation of a Mucoadhesive Vaginal Delivery System for Progesterone," Journal of Controlled Release, Vol. 77, No. 3, 2001, pp. 323-332. doi:10.1016/S0168-3659(01)00520-X

[13] V. Kale, R. Trivedi and P. Muley, "Proposed Design of a Dissolution Apparatus for Vaginal Formulations Containing Probiotics," Dissolution Technologies, Vol. 15, No. 2, 2008.

[14] P. Rege, V. Vilivalam and C. Collins, "Development in Release Testing of Topical Dosage Forms: Use of Enhancer Cell with Automated Sampling," Journal of Pharmaceutical and Biomedical Analysis, Vol. 17, No. 8, 1998, pp. 1225-1233. doi:10.1016/S0731-7085(97)00184-2

[15] W. Liebenberg, E. Engelbrecht and A. Wessels, "A Comparative Study of the Release of Active Ingredients from Semisolid Cosmeceuticals Measured with Franz, Enhancer or Flow through Cell Diffusion Apparatus," Journal of Food and Drug Analysis, Vol. 12, No. 1, 2004, pp. 19-28.
[16] J. Nuttall, D. Thake and M. Lewis, "Concentrations of Dapivirine in the Rhesus Macaque and Rabbit following Once Daily Intravaginal Administration of a Gel Formulation of [14C] Dapivirine for 7 Days," Antimicrobial Agents and Chemotherapy, Vol. 52, No. 3, 2008, pp. 909-914. doi:10.1128/AAC.00330-07

[17] K. Vermani and S. Garg, "The Scope and Potential of Vaginal Drug Delivery," Pharmaceutical Science \& Technology Today, Vol. 3, No. 10, 2000, pp. 359-364. doi:10.1016/S1461-5347(00)00296-0

[18] D. Owen and D. Katz, "A Vaginal Fluid Stimulant," Contraception, Vol. 59, No. 2, 1999, pp. 91-95.

[19] A. Woolfson, R. Malcom and R. Morrow, "Intravaginal Ring Delivery of the Reverse Transcriptase Inhibitor TMC 120 as an HIV Microbicide," International Journal of Pharmaceutics, Vol. 325, No. 1-2, 2006, pp. 82-89. doi:10.1016/j.ijpharm.2006.06.026

[20] K. Gupta, S. Pearce and A. Poursaid, "Polyurethane Intravaginal Ring for Controlled Delivery of Dapivirine, a Nonnucleoside Reverse Transcriptase Inhibitor of HIV-1," Journal of Pharmaceutical Sciences, Vol. 97, No. 10, 2008, pp. 4228-4239. doi:10.1002/jps.21331 